\title{
Point group identification algorithm in dynamic response analysis of nonlinear stochastic systems

\author{
Tao $\mathrm{Li}^{1,2}$, Jian-bing $\mathrm{Chen}^{2}$, Jie $\mathrm{Li}^{2}$
}

1. School of Environment and Architecture, University of Shanghai for Science and Technology, 516 Jungong Road, 200093, Shanghai, China

2. School of Civil Engineering, Tongji University, Siping Road 1239, 200092, Shanghai, China

\begin{abstract}
The point group identification (PGI) algorithm is proposed to determine the representative point sets in response analysis of nonlinear stochastic dynamic systems. The PGI algorithm is employed to identify point groups and their feature points in an initial point set by combining subspace clustering analysis and the graph theory. Further, the representative point set of the random-variate space is determined according to the minimum generalized F-discrepancy. The dynamic responses obtained by incorporating the algorithm PGI into the probability density evolution method (PDEM) are compared with those by the Monte Carlo simulation method. The investigations indicate that the proposed method can reduce the number of the representative points, lower the generalized $F$-discrepancy of the representative point set, and also ensure the accuracy of stochastic structural dynamic analysis.
\end{abstract}

Keywords: non-linear stochastic systems, dynamic response analysis, point group identification algorithm, random-variate space, probability density evolution method, clustering analysis

\section{Introduction}

Stochastic dynamics has gained increasing attention from researchers in many scientific fields. The randomness of stochastic dynamical systems comes from external excitations as well as structural properties [1]. Structural systems are generally at non-linear state when they are subjected to severe external excitations [2]. Due to the coupling of randomness and nonlinearity, the dynamic response of a stochastic system is a random variable or a random process. The more effective method is to capture the probabilistic information of structural dynamic responses [3]. The methods of moment and solving the FPK equation were developed extensively in the field of random vibration. The dynamic responses of a linear stochastic system can be attained by the moment method [4, 5]. But the responses of non-linear stochastic systems cannot be investigated conveniently by this approach because of the non-closed problem of the moment equations $[5,6]$. While the Monte Carlo simulation is always concerned because of its versatility, it is seldom applied in practical large engineering structures due to its prohibitive computational burdens. To solve the problem of compound random vibration involving the randomness from structural properties and external excitations, the Monte Carlo simulation and generalized chaos polynomial method are employed generally [7-9]. Combining the random collocation point method, the investigation of a stochastic dynamic system can be achieved by using the generalized chaos polynomial method [10, 11]. Nevertheless, the number of the orthogonal points of the polynomial grows exponentially with 
the increase of the number of random variables. In the situation, the orthogonal chaos polynomial method is not of acceptable efficiency, namely, it also needs to face the performance impact of the dimensionality curse.

In the past nearly ten years, a new approach named the probability density evolution method (PDEM) proposed by $\mathrm{Li}$ and Chen has been developed systematically [12-16]. It could capture the instantaneous probability density function (PDF) of responses of general non-linear MDOF structures with the randomness involved in structural properties and external excitations. In the PDEM, a generalized density evolution equation (GDEE) is derived and can be solved by the numerical method. The first step to solve the GDEE is the selection of representative point set based on the partition of probability-assigned space [17]. In the PDEM, a representative point set could be picked out by the Grid-type point method or tangent sphere method, if the number of random variables is small $[18,19]$. Otherwise a representative point set could be selected by using the number theoretical method, quasi-symmetric point method and the crude Monte Carlo [18, 20], and 21]. Because of the sparsity and symmetry of points selected by the quasi-symmetric point method, the projections of these points on each dimension of random-variate space overlap each other seriously. Therefore, the information of marginal probability density of random variables cannot be reflected adequately [22]. In the number theoretical method, the representative points were selected further by a hyper-sphere. The points inside the hyper-sphere are picked out and the rest are rejected. Although the number of representative points is reduced effectively, the calculation error increases with the dimensionality of random-variate space increasing. Although the number of representative points selected by the number theoretic method is far less than that needed by the stochastic random number method, as presented in [18], it must be large enough to ensure the computational accuracy of stochastic dynamic analysis.

In the paper, the point group identification (PGI) algorithm is proposed. This algorithm combines the ideas of subspace clustering techniques, e.g. the technique of clustering in quest (CLIQUE) [23], and the graph theory. Clustering techniques have been studied extensively in statistics, pattern recognition, bioengineering and data analysis [24-26]. Based on the subspace clustering techniques and graph theory, this algorithm can identify point groups in the random-variate space and determine the feature points to constitute the representative point set. Each feature point carries the information of all the points in an identified point group represented by it. The probability density solutions of the dynamic responses of stochastic structures in which the randomness come both from structural parameters and external excitation can be attained by incorporating the PGI algorithm into the PDEM. The efficiency and accuracy of the proposed approach is verified through the numerical examples.

We first provide a brief review of the PDEM in Sec. 2 which is the foundation of the algorithm PGI. In Sec. 3 the GF-discrepancy is introduced, which is used as a metric of the homogeneity of a point set in the paper. The efficiency of the algorithm PGI can be verified by the GF-discrepancy. 
The algorithm PGI is proposed in Sec. 4. The numerical examples are adopted to demonstrate the efficiency and accuracy of the algorithm PGI in Sec. 5. In Sec. 6 we conclude by discussing the advantages of the algorithm PGI and an outlook of the further work.

\section{Probability density evolution method}

Considering a generic stochastic dynamical system, the equation of motion is as follows [27]:

$$
\dot{\mathbf{Y}}=\boldsymbol{G}(\Theta, \mathbf{Y}, t)
$$

where $\mathbf{Y}=\left(\mathrm{Y}_{1}, \mathrm{Y}_{2}, \ldots, \mathrm{Y}_{n}\right)$ is a state vector including displacements and velocities of the dynamic system; $\mathbf{Y}_{0}$ is the initial vector; $n$ is the dimension of the dynamical system; $\Theta=\left(\Theta_{1}, \Theta_{2}, \ldots, \Theta_{s}\right)$ is an $s$-dimensional random vector involved with known joint probability density function $p_{\Theta}(\boldsymbol{\theta})$. $\Theta_{j}(j=1,2, \ldots, s)$ are random variables representing the randomness either from excitations or system parameters. In some cases, random excitations or random system parameters are modeled as random processes, which can be further expressed by a combination of a series of random variables, say through some decomposition methods of random process, e.g. the Karhunen-Loeve decomposition method [34] and orthogonal decomposition method [27]. Namely, the random process should firstly be represented by some random variables. Then these random variables together with other random variables become the components of the random vector $\Theta . \Theta$ is still a random vector consisting of random variables, but the dimensionality of the random vector $\Theta$ increases.

If a random-variate space $\Omega_{\Theta}$ is divided into some non-overlapping partitioning subspace $\Omega_{q}, q=1,2, \ldots, \mathrm{n}_{\mathrm{pt}}$, and

$$
P_{q}=\int_{\Omega_{q}} p_{\Theta}(\boldsymbol{\theta}) d \boldsymbol{\theta}
$$

is the assigned probability over $\Omega_{q}[21]$. The advanced probability density evolution equation is proposed [16]:

$$
\frac{\partial p_{q}(y, t)}{\partial t}+\int_{\Omega_{q}}\left(\dot{\mathrm{Y}}(\boldsymbol{\theta}, t) \frac{\partial p_{\mathrm{Y} \Theta}(y, \boldsymbol{\theta}, t)}{\partial y}\right) \mathrm{d} \boldsymbol{\theta}=0
$$

where $p_{\mathbf{Y} \Theta}(y, \boldsymbol{\theta}, t)$ is the joint probability density function of $(\mathbf{Y}(t), \boldsymbol{\Theta})$. It is denoted that:

$$
p_{q}(y, t)=\int_{\Omega_{q}} p_{\mathrm{Y \Theta}}(y, \boldsymbol{\theta}, t) \mathrm{d} \boldsymbol{\theta}, q=1,2, \ldots, \mathrm{n}_{\mathrm{pt}}
$$

where functions $p_{q}(y, t), q=1,2, \ldots, \mathrm{n}_{\mathrm{pt}}$, are not probability density functions because $\int_{-\infty}^{+\infty} p_{q}(y, t) d y=P_{q} \neq 1$. However, after defining a normalized function $\tilde{p}_{q}(y, t)=p_{q}(y, t) / P_{q}$, the consistency condition is satisfied: $\int_{-\infty}^{+\infty} \tilde{p}_{q}(y, t) d y=1$. The function $\tilde{p}_{q}(y, t)$ is called the partial-probability density function over $\Omega_{q}$; and it is completely governed by Eq. (3) once a partition of probability-assigned space is determined [16].

The solution of $p_{q}(y, t)$ can be obtained by solving Eq. (3), if the velocity information of all the 
points in $\Omega_{q}$ is obtained. But this condition is very difficult to be satisfied. Generally, a representative point $\theta_{q}$ is used to represent the information of all points in the subspace $\Omega_{q}$, namely:

$$
\dot{\mathrm{Y}}(\boldsymbol{\theta}, t) \doteq \dot{\mathrm{Y}}\left(\boldsymbol{\theta}_{q}, t\right)
$$

Now Eq. (3) is equivalent to following equation [16]:

$$
\frac{\partial p_{q}(y, t)}{\partial t}+\dot{\mathrm{Y}}\left(\boldsymbol{\theta}_{q}, t\right) \frac{\partial p_{q}(y, t)}{\partial y}=0
$$

It is noticed that

$$
\begin{aligned}
p_{\mathbf{Y}}(y, t) & =\int_{\Omega_{\Theta_{\boldsymbol{\theta}}}} p_{\mathrm{Y} \boldsymbol{\Theta}}(y, \boldsymbol{\theta}, t) d \boldsymbol{\theta} \\
& =\sum_{\mathrm{n}=1}^{\mathbf{n}_{\mathrm{pt}}} p_{q}(y, t)=\sum_{\mathrm{n}=1}^{\mathrm{n}_{\mathrm{pt}}} P_{q} \cdot \tilde{p}_{q}(y, t)
\end{aligned}
$$

The PDF of $\mathbf{Y}(t)$ can be obtained by solving the partial differential Eq. (6) and Eq. (7).

From the above details, it is seen that the selection of representative points $\theta_{q}, q=1,2, \ldots, \mathrm{n}_{\mathrm{pt}}$, plays a very important role in the PDEM, it directly influences the computational accuracy of the PDEM.

\section{Generalized F-discrepancy of a point set}

Considering a point set $\mathcal{P}_{n}$ with $n$ points in a $k$-dimensional hypercube $C^{k}=[0,1]^{k}$, the discrepancy of the point set is defined as follows [28]:

$$
D\left(\mathcal{P}_{n}\right)=\sup _{\boldsymbol{x} \in C^{k}}\left|\frac{N\left(\mathcal{P}_{n},[\mathbf{0}, \boldsymbol{x})\right)}{n}-V([\mathbf{0}, \boldsymbol{x}))\right|
$$

where $N\left(\mathcal{P}_{n},[\mathbf{0}, \boldsymbol{x})\right)$ is the number of points lying inside the high-dimensional hypercube $[\mathbf{0}, \boldsymbol{x})$, $V([\mathbf{0}, \boldsymbol{x}))=\prod_{j=1}^{k}{ }_{j}$ is the volume of the high-dimensional hypercube $[\mathbf{0}, \boldsymbol{x})$.

The Koksma-Hlawka inequality states that the error of numerical integration is bounded by the product of the discrepancy of the point set $\mathcal{P}_{n}$ and the total variation of the function, i.e. [28]:

$$
\left|\int_{C^{k}} f(\boldsymbol{x}) \mathrm{d} \boldsymbol{x}-\frac{1}{n} \sum_{q=1}^{n} f\left(\boldsymbol{x}_{q}\right)\right| \leq D\left(\mathcal{P}_{n}\right) \cdot T V(f)
$$

where $D\left(\mathcal{P}_{n}\right)$ is the discrepancy of the point set $\mathcal{P}_{n}, T V(f)$ is the total variation of the function, which reflects the irregularity of the function $f(x)$.

The above equation indicates that the accuracy of numerical integration may be improved by decreasing the discrepancy of the point set as long as the function $f(x)$ has the bounded variation [22].

A new discrepancy called the generalized F-discrepancy (GF-discrepancy) is proposed by Chen and Zhang in 2013. This discrepancy may be employed to measure the quality of a point set. It is defined as follows [29]:

$$
D_{\mathrm{GF}}\left(\mathcal{P}_{n}\right)=\max _{1 \leq i \leq s}\left\{\sup _{x \in R}\left|F_{n, i}(x)-F_{i}(x)\right|\right\}
$$

where $F_{i}(x)$ is the marginal cumulative distribution function of the $i$ th random variable $X_{i}, F_{n, i}(x)$ is 
the empirical cumulative distribution function of the $i$ th random variable $X_{i} \cdot F_{n, i}(x)$ is defined as [29]:

$$
F_{n, i}(x)=\sum_{q=1}^{n} P_{q} \cdot I\left\{x_{q, i} \leq x\right\}
$$

where $x_{q, i}$ is the $i$ th component of the point $\boldsymbol{x}_{q}, P_{q}$ is the assigned probability accordingly.

The marginal discrepancy of the point set on each dimension is proposed as follows [29]:

$$
D_{\mathrm{F}, i}\left(\mathcal{P}_{n}\right)=\sup _{x \in R}\left|F_{n, i}(x)-F_{i}(x)\right|
$$

The GF-discrepancy can be interpreted as the maximum of all marginal discrepancies.

\section{Point group identification algorithm}

Points in the same point group have great similarity with each other because of the similarity of their spatial positions or other special natures. The point group analysis (PGI) algorithm proposed in the paper can be employed to identify point groups and determine feature points of point groups. In the algorithm PGI, the point groups are defined as follows:

Definition 1 For an arbitrary given point set $S$ in a random-variate space $\Omega_{\Theta}$, which is divided into some non-overlapping subspaces $\Omega_{q}$, namely: $\Omega_{i} \cap \Omega_{j}=\varnothing, \forall i \neq j, \cup_{1}^{\mathrm{n}_{\mathrm{pl}}} \Omega_{q}=\Omega_{\Theta}$, the point groups $G_{q}$ are constituted by all the points in $\Omega_{q}, q=1,2, \ldots, \mathrm{n}_{\mathrm{pt}}$.

Based on a certain criterion, the similar points in an initial point set can be identified as a point group by the algorithm PGI. The algorithm can determine the feature points (representing the groups) and the isolated points (no similarity with other points). The feature points and isolated points form the final representative point set.

Each dimension of the random-variate space is partitioned into the identical number of equal length intervals, and the number of points lying inside each unit of partitioning is found. Each unit has the identical volume, so the density of the unit can be approximated by the number of the points inside each unit. A unit is called dense unit only if the density of points inside it is more than a given threshold. To identify point groups, firstly all dense units in each dimensional subspace are found. The dense units connecting to each other form a region. All points in the same region constitute a point group. Finally, the feature points of all point groups and isolated points are gathered into a final representative point set.

The algorithm PGI is carried out with the following three steps.

Step 1: Identify dense units.

Step 2: Judge connectivity of the dense units and identify point groups.

Step 3: Determine feature points and isolated points to form the final representative point set.

\subsection{Identifying dense units}

In general, the similarity of data objects could be measured in a low-dimensional space by $L_{k}$ norm $\left(x, y \in \Re^{m}, k \in N, L_{k}=\sum_{i=1}^{m}\left(\left\|x_{i}-y_{i}\right\|^{k}\right)^{1 / k}\right)$. A distance metric function $\operatorname{dist}_{k}^{m}(x, y)$ is used to express the distance between $\left(x_{1}, \ldots, x_{m}\right)$ and $\left(y_{1}, \ldots, y_{m}\right)$ based on $L_{k}$ norm [30]: 


$$
\operatorname{dist}_{k}^{m}(x, y)=\left[\sum_{i=1}^{m}\left(x_{i}-y_{i}\right)^{k}\right]^{1 / k}
$$

where $x_{i}$ is the projection of the point $x$ on the $i$ th dimension, $y_{i}$ is the projection of the point $y$ on the $i$ th dimension, $m$ is the dimensionality of the data space.

Theorem [30]: Let $H$ be the arbitrary distribution of $n$ points, then:

$$
C_{k} \leq \lim _{m \rightarrow \infty} E\left[\frac{\left(\operatorname{Dmax}_{m}-\text { Dmin }_{m}\right)}{m^{1 / k^{-1} / 2}}\right] \leq(n-1) C_{k}
$$

where $\operatorname{Dmax}_{m}$ and $\operatorname{Dmin}_{m}$ are the farthest and nearest distance of $n$ points to the origin by the distance metric based on $L_{k}$ norm respectively, $C_{k}$ is some constant dependent on $k$.

Proof: See [30] for proof of a more detailed version of this result.

The above theorem shows that in a high-dimensional data space $\operatorname{Dmax}_{m}-\operatorname{Dmin}_{m}$ converges at the rate of $m^{1 / k^{-1 / 2}}$, independent of the data distribution. Moreover, the convergence is faster when the value of $k$ of the $L_{k}$ metric is greater (it is noticed that $1 / k<1 / 2$ if $k>2$ ).

Because the $L_{k}$ norm converges in a high-dimensional space, it is very difficult to find points with similarity in the original space. Generally, a stochastic dynamic system contains many random variables. These random variables expand to a high-dimensional random-variate space. The similarity of data objects could be measured by a density-based approach. The density of points is defined as the percentage of the number of points in a unit generated by partitioning the data space among the number of points in total data space. A unit is defined as a dense unit when the density of points lying inside it is greater than a given threshold. The following steps are carried out to find all dense units sequentially in each different dimensional subspace.

(1) Choose an initial representative point set which follows a certain distribution according to the dimensionality of the random-variate space.

(2) Partition each dimension of the data space into $\xi$ intervals of equal length, and choose the unit in which one or more points are contained as a computing unit.

(3) Identify all dense units in the different subspaces by the Apriori algorithm [37] base on a given density threshold $\tau$.

(4) Choose the subspace that contains point groups.

If each dimension of the data space is numbered sequentially, only the first sequential $s$-dimensional subspace can be selected as the basis for the following work. For example, each dimension of a 3-dimensional space is numbered with dimension 1, 2, and 3. The 2-dimensional subspace of this 3-dimensional space is composed of dimension 1 and 2, or dimension 2 and 3, or dimension 1 and 3. Only the 2-dimensional subspace formed by dimension 1 and 2 could be chosen. This means that the random variables should be sorted according to their importance in a stochastic dynamical system. So the 
workload related to data processing could be reduced effectively through step (4).

The techniques used in step (2) and (3) are based on an automatic subspace clustering algorithm (CLIQUE algorithm) proposed by Agrawal et al. in 1998 [26]. The feasibility of the step (4) is illustrated as follows.

For instance, a seismic stochastic process in a stochastic dynamic system can be expanded as follows based on the orthogonal expansion method [31]:

$$
\ddot{X}(t)=\sqrt{2} \sum_{j=1}^{r} \sqrt{\lambda_{j}} \xi_{j} f_{j}(t)
$$

where

$$
f_{j}(t)=\sqrt{2} \sum_{k=1}^{N} \chi_{k+1} \varphi_{j k} \ddot{\phi}_{k}(t)=-\sum_{k=1}^{N}\left(\frac{2 k \pi}{T_{s}}\right)^{2} \chi_{k+1} \varphi_{j k} \phi_{k}(t)
$$

where $\ddot{X}(t)$ is the acceleration of the ground motion; $\lambda_{j}$ and $\varphi_{j}$ are the eigenvalue and standard eigenvector of the covariance matrix $R$ respectively, $R$ is the covariance matrix of the seismic stochastic process; $\varphi_{j k}$ is the $k$ th element of $\varphi_{j},\left\{\xi_{1}, \xi_{2}, \ldots, \xi_{r}\right\}$ is a set of mutually independent standard Gaussian random variables, $\phi_{k}(t)$ is a set of normalized Hartley orthogonal basis $[\mathbf{3 8 , 4 0 ]}$, $\chi_{k+1}(k=1,2, \ldots, N)$ is a set of coefficients to make up the truncated error and obtained by the principle of energy equivalence [31], $r$ is the number of random variables determined by the orders of the first several eigenvalues.

The details of orthogonal decomposition method and Hartley orthogonal basis are introduced comprehensively in $[27,38,40]$. The procedures of determination of $\chi_{k+1}$ based on the principle of energy equivalence could be found in [31]. The premise of Eqs. (15)-(16) is the solution of the eigenvalues of the correlation matrix $R$. If the correlation matrix $R$ of the seismic stochastic process is given, its eigenvalues could be obtained conveniently by solving the characteristic equation of the covariance matrix $R$. The accelerations of the ground motion could be attained according to Eqs. (15)-(16). Here the results of the eigenvalues of the covariance matrix $R$ in reference [31] (as shown in Fig. 1) are employed to illustrate why the random variables should be sorted according to their importance in the stochastic dynamic analysis. It is found from Eq. (15) that the number and the permutation of the random variables depend on those of the eigenvalues of the covariance matrix $R$. The effects on Eq. (15) of the former random variables are larger than that of the latter random variables. So the subspace expended by the former random variables carries more information. This is the reason that only the first $s$-dimensional subspace is chosen in step (4).

Fig. 1 presents a trend of eigenvalues changing with their orders. 


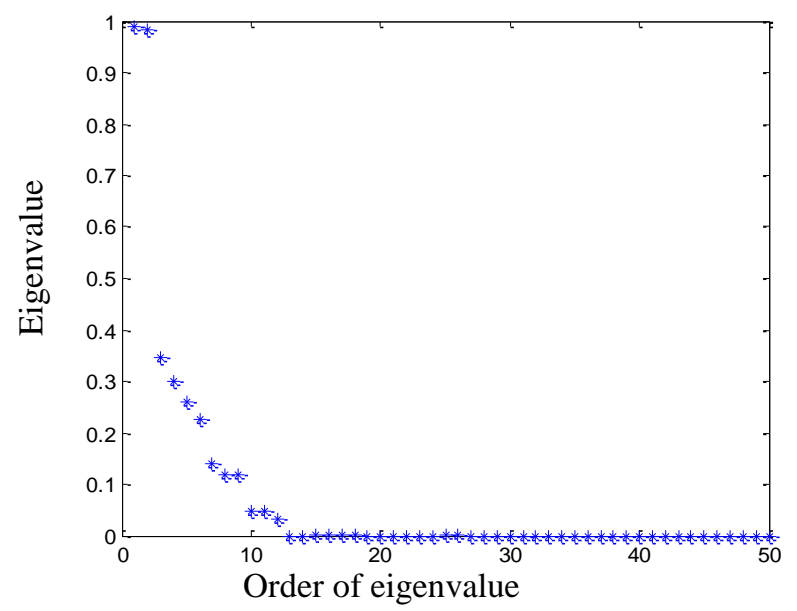

Fig. 1 First 50 eigenvalues of the correlation matrix [31]

Fig. 1 shows that the eigenvalue decreases as the order of eigenvalue increasing. The first $r$ eigenvalues are chosen to solve Eq. (15) and Eq. (16). Correspondingly, there are $r$ random variables determined. These random variables expand an $r$-dimensional space. While sometimes point groups could not be identified in the original random-variate space, they could be identified in some subspaces of the original space. The subspace expanded by the first several random variables is chosen in order to reduce errors, because the influence from the preceding eigenvalues is greater than it from the latter eigenvalues. This is the reason that the subspace is chosen according to step (4).

Now all dense units can be found via the above method. The following step is to identify all point groups in the random variable space.

\subsection{Identifying point group}

Let $\quad \boldsymbol{G}=\langle\boldsymbol{V}(\boldsymbol{G}), \boldsymbol{E}(\boldsymbol{G}), \varphi(\boldsymbol{G})\rangle \quad$ be a graph composed by vertex set $\boldsymbol{V}(\boldsymbol{G})=\left\{v_{1}, v_{2}, \ldots, v_{n}\right\},(\boldsymbol{V}(\boldsymbol{G}) \neq \phi) \quad$, edge $\quad$ set $\quad \boldsymbol{E}(\boldsymbol{G})=\left\{e_{1}, e_{2}, \ldots, e_{m}\right\}$ and incidence function $\varphi(\boldsymbol{G})=\boldsymbol{E} \rightarrow \boldsymbol{V} \times \boldsymbol{V}$. All the dense units identified in an $s$-dimensional subspace via the above techniques can be seen as the vertex set $V$ of the graph $G$.

\subsubsection{Identifying point groups in total space}

Point groups can be identified by the method of judging connected graphs. Calculate the adjacent matrix $\boldsymbol{A}=\left(a_{i j}\right)$ of the graph $\boldsymbol{G}$, where $a_{i j}$ is the number of edges that start with the vertex $v_{i}$ and end with the vertex $v_{j}$. If $a_{i j} \neq 0$, it means that there are edges between the vertex $v_{i}$ and $v_{j}$. Define a block containing all units which have edges between themselves and $v_{i}$ or $v_{j}$ and number it. Then each $a_{i j}$ is searched, and all different blocks in the $s$-dimensional subspace are found. All the points lying inside a same block constitute a point group. These blocks can be seen as the equivalence of connected graphs. 


\subsubsection{Identifying point groups in the subspace}

With the dimensionality increasing, it becomes difficult to identify point groups in the original data space, but point groups can be found in some $s$-dimensional subspaces. This conclusion is expounded in section 4.1. Lots of isolated points are found with appearance of point groups identified in the subspaces. If the number of isolated points is very great, the number of representative points is also great because the representative point set is constituted by feature points and isolated points. The number of representative points can be reduced by defining some auxiliary blocks.

(1) Identifying new point groups using auxiliary blocks

The key factors influencing the number of point groups are the number of intervals $\xi$ and the density threshold $\tau$ introduced in section 4.1. The blocks (connected dense units) are composed of the units that the densities of points inside them are greater than the threshold $\tau$. In order to reduce the number of the representative points, we try to find the connected units in those units that the densities of points inside them are lower than the threshold $\tau$. These connected units can be defined as auxiliary block. The points in an auxiliary block also constitute a point group.

The number of the representative points is reduced effectively by the auxiliary blocks, but the $G F$-discrepancy of the representative point set is increased. This means that the accuracy of identifying the point groups is reduced.

(2) Improving the accuracy of identifying point groups

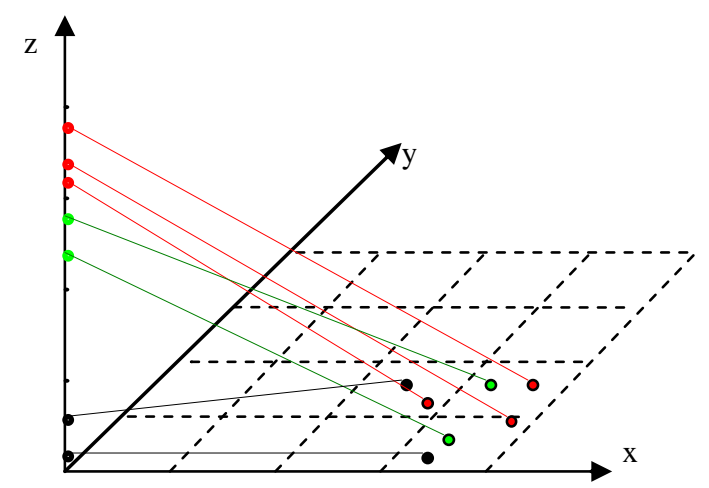

Fig. 2 Projection of points in the remaining

1-dimensional subspace

The dense units are identified in an $s$-dimensional subspace of the $k$-dimensional original random-variate space. Then point groups are identified in this $s$-dimensional subspace. The influence of the remaining $(k-s)$-dimensional subspace is neglected. While the first $s$-dimensional projections of points are in the same block, it is possible that their latter $(k-s)$-dimensional projections do not lie inside a same region of the remaining $(k-s)$-dimensional subspace. Each dimension of the $k$-dimensional space 
is partitioned into identical number of equal length intervals in section 4.1. Each dimension of the remaining $(k-s)$-dimensional subspace is also partitioned, i.e., the remaining $(k-s)$-dimensional subspace is also partitioned into some units with identical volume. An additional condition on the definition of the point group is imposed. This condition is the projections of points in the remaining $(k-$ $s$ )-dimensional subspace should lie inside the same remaining $(k-s)$-dimensional unit. This condition is called the restraint condition of the remaining subspaces. Fig. 2 is the scattering sketch for the projections of points in the remaining 1-dimensional subspace.

As shown in Fig. 2, the original random space is a 3-dimensional space; a block (the connected units) is found in the 2-dimensional subspace. There are 7 points inside it. If we do not add any condition, these 7 points inside the same block can be indentified as a point group. However, Fig. 2 shows that the projections of these 7 points in the remaining 1-dimensional space scatter in three different regions (units). The points with their latter $(k-s)$-dimensional projections inside same region (unit) of the remaining subspace have greater similarity with each other because of the similarity of their spatial positions. They belong to the same point group. As presented in Fig. 2, the red points, the green points and black points constitute different point groups respectively.

Interestingly, although the above technique of handling point groups is simple, the result is satisfactory because the GF-discrepancy is decreased effectively. The computed results are presented in section 5. The accuracy of identifying point groups can be improved by adding the remaining subspace restraint condition.

\subsection{Determining feature points}

To determine the feature point of the point group, the center unit of the connected graph should be found firstly, and the points in the center unit are viewed as the candidates for feature points. The feature points of point groups are finally determined based on the criterion of the minimum $G F$-discrepancy.

\subsubsection{Center of a connected graph}

Different blocks (connected graphs) can be found by using the adjacent matrix $\boldsymbol{A}=\left(a_{i j}\right)$ of a graph $G$ in section 4.2. The determination of the connected graph is a location problem. The centers of blocks are determined according to the method for computing the weighted center of a graph.

Let $F$ be an $n$-order connected graph. $D=\left(d_{i j}\right)_{\mathrm{n} \times \mathrm{n}}$ is its shortest distance matrix, where $d_{i j}$ is the shortest distance among all distances between the vertex $v_{j}$ and other vertexes except the vertex $v_{i}$, $A=\left(a_{1}, a_{2}, \ldots, a_{\mathrm{n}}\right)$ is a weight matrix, where $a_{1}, a_{2}, \ldots, a_{\mathrm{n}}$ are weights of each vertex respectively. The weight distance between the vertex $v_{i}$ and other vertex is presented as follows [32]:

$$
d_{i}=\sum_{j=1}^{n} a_{j} d_{i j}, \quad i=1,2, \ldots, \mathrm{n}
$$

It is denoted that:

$$
i_{0}=\min _{1 \leq i \leq \mathrm{n}}\left\{d_{i}\right\}
$$


The vertex corresponding to $i_{0}$ is the center of the connected graph $F$. The vertex is the center unit found in the connected graph.

\subsubsection{Feature points of point groups}

The points in the above center units are chosen as the candidates for feature points of point groups. If there are 2 or more than 2 candidates in a point group, the point with greatest weight is chosen as the candidate for the feature point of this group.

GF-discrepancy can be used to estimate the uniformity of the point set. To make the point set more uniform, the point which makes the marginal discrepancy of the point set minimum is chosen as the feature point (as shown in Eq. 10).

The coordinate of the point is adjusted as follows [22]:

$$
\begin{gathered}
x_{s, i}=F_{i}^{-1}\left(\sum_{q=1}^{n} P_{q} \cdot I\left\{x_{q, i} \leq x_{s, i}\right\}-\frac{1}{2} P_{s}\right) \\
\mathrm{s}=1,2, \cdots, n ; i=1,2, \cdots, m
\end{gathered}
$$

where $x_{s, i}$ is the coordinate of the point, $i$ is the mark of the dimension on which the coordinate of the point is adjusted, $s$ is the position code of the point on the $i$ th dimension, $F_{i}^{-1}$ is the inverse cumulative distribution function, $P_{q}$ is the assigned probability of each point, $I\{\cdot\}$ is the indication function: if the event in the bracket is true, $I\{\cdot\}=1$, otherwise, $I\{\cdot\}=0, P_{s}$ is the assigned probability of the No. $s$ point.

The adjusted point is determined as the feature point of the point group.

If there are still some isolated points in the random-variate space, these isolated points and feature points constitute the final representative point set together.

\subsubsection{Assigned probability of representative point}

The assigned probability of each representative point is the sum of the original assigned probabilities of all points in a point group. This means that the representative points can carry the information of all the points in the original point set in a degree.

4.4. Procedures of investigation of stochastic dynamical systems by using the PGI algorithm incorporated into PDEM

The probability density solution of the stochastic dynamic responses of a nonlinear structure could be obtained conveniently by using the PGI algorithm incorporated into the PDEM. The solving procedure is presented as follows:

Part I: Determine the representative points of the random-variate space by PGI algorithm

- Establish the stochastic equation of motion of the stochastic dynamical system.

- Determine the number of the random variables of the stochastic dynamical system.

- Choose an initial point set of the random-variate space expanded by the above random variables. 
- Find the point groups in the initial point set and determine the feature points $\left(\boldsymbol{\theta}_{q}, q=1,2, \ldots, \mathrm{n}_{p t}\right)$ to form the representative point set by using the PGI algorithm (as shown in Sec. 4.1 to Sec. 4.3).

Part II: Attain the probability density solution of the dynamic responses of the nonlinear stochastic dynamical system

- Take representative point $\theta_{q}$ starting at $q=1$ and let $q=q+1$ each time the loop is taken.

- Carry out the deterministic dynamic analysis of Eq. (1) for $\Theta=\theta_{q}$ (as shown in Sec.2).

- Solve Eq. (6) by some numerical methods, such as the finite difference method proposed reference [15].

- $\quad$ Take numerical integration in Eq. (7).

- $\quad$ Repeat the above steps until $q=\mathrm{n}_{p t}$.

Because of the reduction of the number of the representative points by using the algorithm PGI, the calculation efficiency of the PDEM is improved greatly without losing the calculation accuracy. In Sec. 5 a numerical example is taken to illustrate the effectiveness and the accuracy of the PGI algorithm.

\section{Result analysis}

\subsection{2-dimensional random-variate space}

An example of identifying point groups in a 2-dimentional random-variate space is taken to illustrate the feasibility of the algorithm PGI. The algorithm PGI should be carried out according to the three steps summarized in Sec. 4. The initial point set consisting of 80 points could be generated by the pseudo random number generator.

Fig. 3 shows the scattering of 80 points in a 2-dimensional random-variate space generated by the pseudo random number generator. Regions bounded by the blue lines are the Voronoi regions [39] represented by these points. The initial point set constituted by these 80 points can be called PRN_80. The GF-discrepancy of the PRN_80 point set is 0.0861 .

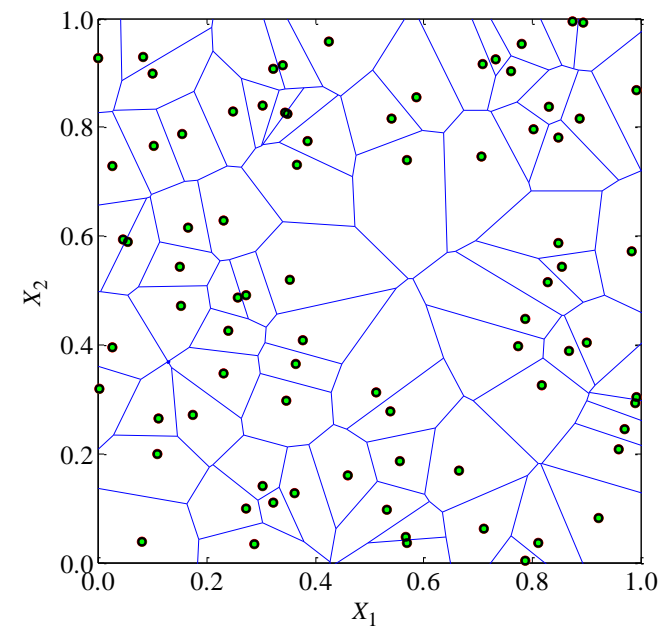

Fig. 3 Scattering of 80 points generated by pseudo random number method

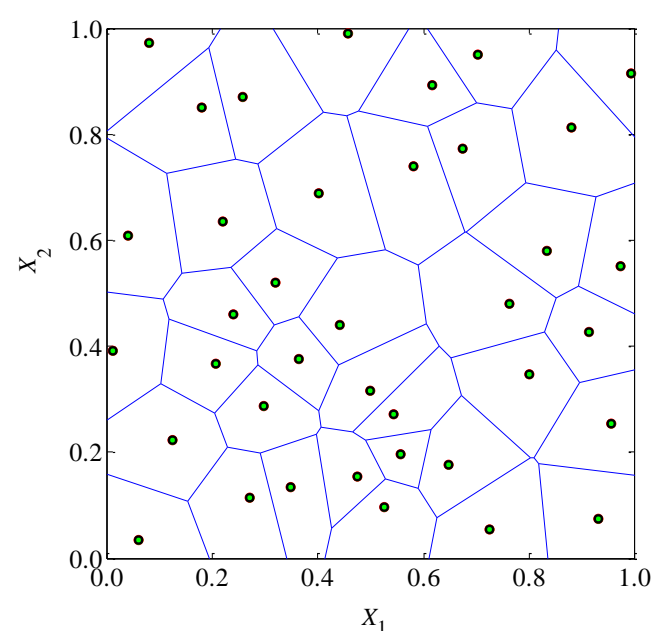

Fig. 4 Scattering of 38 representative points generated by the PGI algorithm 


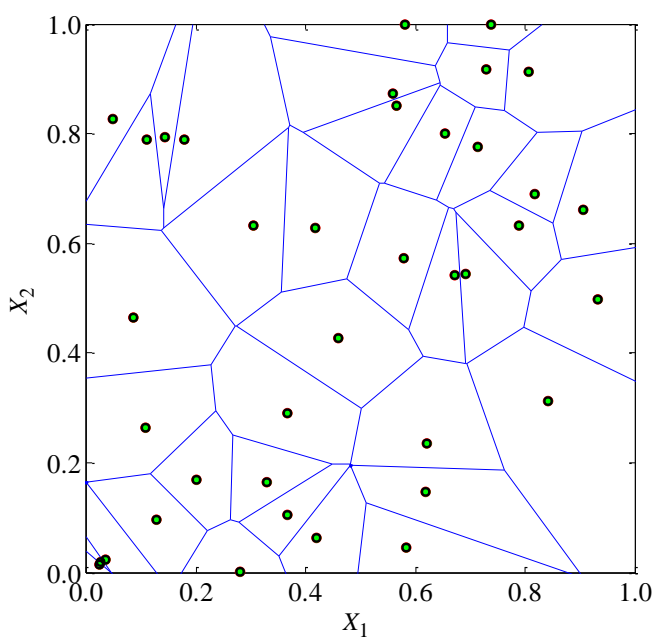

Fig. 5 Scattering of 38 points generated by pseudo random number method

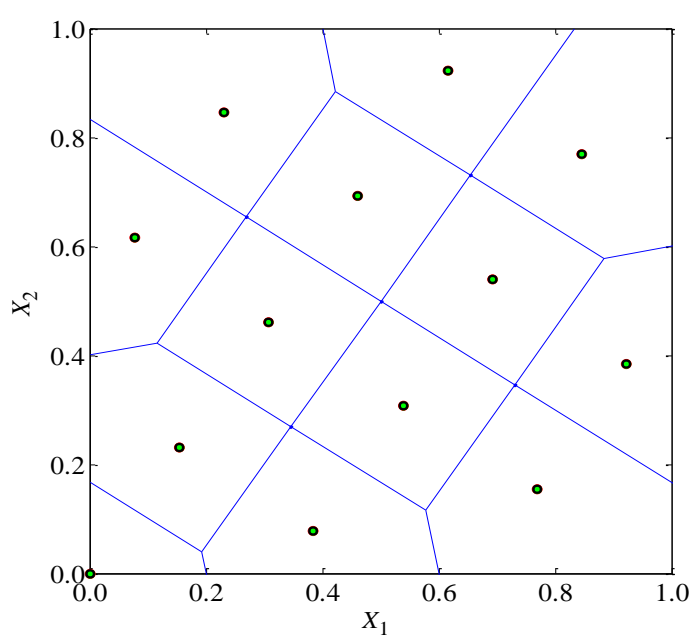

Fig. 6 Scattering of the points in the 2-dimensional number theoretical point set

Each dimension is partitioned into 10 intervals $(\xi=10$ ). The density threshold $\tau$ is 0.025 .23 point groups can be identified in the original space by PGI algorithm. Accordingly, 23 feature points and 15 isolated points are determined as the representative points (the point set constituted by the above points is called PGI point set). The scattering of 38 representative points is shown in Fig. 4. The GF-discrepancy of the PGI point set is 0.0438 . In order to indicate the effectiveness of the PGI algorithm, 38 points are generated directly by pseudo random number method. PRN_38 point set represents the point set formed by 38 pseudo random numbers. The scattering of 38 pseudo random numbers is shown in Fig.5. The GF-discrepancy of PRN_38 point set is 0.0925, which is much greater than the GF-discrepancy of the PGI point set. The details of three kinds point sets are presented in Table 1.

Table 1 Discrepancies of three point sets in the 2-dimensional space

\begin{tabular}{ccccc}
\hline Case & Item & $\begin{array}{c}\text { PRN_80 } \\
\text { point set }\end{array}$ & $\begin{array}{c}\text { PGI } \\
\text { point set }\end{array}$ & $\begin{array}{c}\text { PRN_38 } \\
\text { point set }\end{array}$ \\
\hline $\begin{array}{c}\text { 2-dimensional } \\
\text { space }\end{array}$ & Point number & 80 & 38 & 38 \\
& GF-discrepancy & 0.0861 & 0.0438 & 0.0925 \\
\hline
\end{tabular}

Seen from Fig. 4 and Fig. 5, the distribution of 38 representative points in the PGI point set is more uniform than the distribution of 38 points in the PRN_38. It is found from Fig. 4 and Fig. 3 that the former distribution is also more uniform than the distribution of 80 points in the PRN_80. Seen from Table 1, the $G F$-discrepancy of the PGI point set is less than the one of the PRN_80 point set effectively. Meanwhile, the number of points in the PGI point set is also reduced effectively. It should be noted that the application of the PGI algorithm in a 2-dimensional number theoretical point set has no such obvious effect (although it is feasible), because the points in a 2-dimensional number theoretical point set 
themselves are very uniform, as shown in Fig. 6. The similarity of the points can hardly be found based on the density criterion.

\subsection{0-dimensional random-variate space}

In general, the point set generated by pseudo random number method is not the best choice for us to analyze the responses of a stochastic dynamical system based on the PDEM. This is mainly because of the indeterminacy of the point set generated by the pseudo random number method. The number theoretical point set, Sobol point set, and Halton point set are always employed to represent the random-variate space. In the paper the number theoretical point set is taken as an example to interpret the application of the PGI algorithm in a high-dimensional space. It is known that the number of points in a number theoretical point set increases rapidly with the dimensionality of the random-variate space increasing. To reduce the number of the points, the representative points could be sieved by a hypersphere with a radius of $r$ in the PDEM [18]. As denoted in section 5.1, the distribution of the points generated by the number theoretical method is very uniform in a 2-dimensional plane. But does the distribution of points in the number theoretical point set still keep homogeneous in a high-dimensional space? Interestingly, it is not the truth because the point groups could be identified when the dimensionality of the random-variate space is more than 3 . When the dimensionality of the random-variate space is between 3 and 5, the point groups in the number theoretical point set can be identified in the initial space. But with the increase of the dimensionality of the random-variate space, as same as the general data space, the point groups in the number theoretical point set can not be found. This situation is not because of the uniformity of the number theoretical point set but of the sparsity of the points. However, the point groups can be identified in the subspaces of the original random-variate space by the PGI algorithm. A 10-dimensional number theoretical point set is adopted as an example.

In a 10-dimensional random-variate space the point groups in the original space can not be identified because of the sparsity of dada objects; nevertheless, the points groups are found in the 7-dimensional subspace. There are 13587 points in the original point set determined by the number theoretical method. 6 blocks and 68 auxiliary blocks are found in the 7-dimensional subspace. 247 point groups are indentified through the restraint condition of remaining subspace. Therefore there are 247 feature points in total. These feature points constitute the representative point set of the 10-dimension random-variate space (this point set is called the PGI point set). Its GF-discrepancy is 0.0114 . We can also choose 247 representative points by a hypersphere with the radius of $r=1.212 \lambda$, where $\lambda$ is the value of the boundary (this point set is called NTM point set). The third point set formed by 247 points is generated by pseudo random number method (PRN point set). The GF discrepancies of these three point sets are presented in Table 2.

As shown in Table 2, the number of representative points can be reduced from 13578 to 247 by the PGI algorithm. The GF-discrepancy of the PGI point set is lower obviously than those of the NTM point set and PRN point set. The result shows that the distribution of the points generated by the number theoretical method in high-dimensional space is not homogeneous as those in the 2-dimensional plane. Fewer points in the initial point set are picked out to represent the random-variate space by the PGI algorithm. 
Table 2 Discrepancies of three kinds of point sets in the 10-dimensional space

\begin{tabular}{ccccc}
\hline Case & Item & PGI & NTM & PRN \\
\hline 10-dimesional & Point number & 247 & 248 & 247 \\
space & GF-discrepancy & 0.0114 & 0.1398 & 0.0736 \\
\hline
\end{tabular}

\subsection{Numerical case}

A five-storey and double span shear frame is taken as a numerical case here (shown in Fig. 7). The mean values of the lumped mass of the structure from the bottom storey to the top storey are $2.5 \times 10^{5} \mathrm{~kg}$, $2.5 \times 10^{5} \mathrm{~kg}, 2.2 \times 10^{5} \mathrm{~kg}, 2.2 \times 10^{5} \mathrm{~kg}$ and $2.0 \times 10^{5} \mathrm{~kg}$ respectively. The mean values of the stiffness of the structure from the bottom to the top are $3.7 \times 10^{8} \mathrm{kN} / \mathrm{m}, 3.7 \times 10^{8} \mathrm{kN} / \mathrm{m}, 3.5 \times 10^{8} \mathrm{kN} / \mathrm{m}$, $3.5 \times 10^{8} \mathrm{kN} / \mathrm{m}$ and $3.2 \times 10^{8} \mathrm{kN} / \mathrm{m}$ respectively. Mass and stiffness of the structure follow the Gaussian distribution. There are 10 random variables in total. The coefficients of variation of these random variables are all 0.2. The Bouc-Wen hysteretic model is used to express the relation between the restoring force and the inter-storey drift. Rayleigh damping is employed, i.e. $C=a M+b K_{d}$, where $K_{d}$ is the tangent stiffness matrix, $a=0.3, b=0.0102$. The structure is subjected to earthquake excitation in the shape of the east-west component of the El Centro acceleration record. The displacement of the bottom storey is investigated.

Noting that only the randomness from the structural parameters are involved in this case, the stochastic equation of motion of the five-storey structure subjected to earthquake excitation is as follows

$$
\boldsymbol{M}(\xi) \ddot{\boldsymbol{X}}+\boldsymbol{C}(\xi) \dot{\boldsymbol{X}}+\boldsymbol{R}(\xi, \boldsymbol{X})=-\boldsymbol{M I} \ddot{\boldsymbol{X}}_{g}(t)
$$

where $\ddot{\boldsymbol{X}}, \dot{\boldsymbol{X}}$ and $\boldsymbol{X}$ are the acceleration matrix, velocity matrix and displacement matrix respectively; $\boldsymbol{M}$ and $\boldsymbol{C}$ are the mass and damping matrices respectively; $\boldsymbol{I}=(1,1, \ldots, 1)^{\mathrm{T}}$ is a column vector with all components being 1; $\ddot{X}_{g}(t)$ is the ground motion acceleration given by the El Centro acceleration record; $\xi=\left(\xi_{1}, \xi_{2}, \ldots, \xi_{10}\right)$ is the basic random vector involved in the structural properties representing the randomness coming from the mass and stiffness of five stories; $\boldsymbol{R}(\xi, \boldsymbol{X})=\left(R_{1}, R_{2}, \ldots, R_{5}\right)^{\mathrm{T}}, \quad R_{i}$ is the nonlinear restoring force applied on the node $i(i=1,2, \ldots, 5)$, it can be expressed as follows

$$
R_{i}=\sum_{e=1}^{m_{i}} R_{i}^{e}
$$

where $e$ represents the element connected to the node $i ; R_{i}^{e}$ is the internal restoring force at the node $i$ of the element $e ; m_{i}$ is the number of the elements connected to the node $i$.

According to Wen [35], restoring force $R_{i}^{e}$ could be partitioned into linear part and hysteretic part as follows 


$$
R_{i}^{e}=\alpha k_{i} x_{i}+(1-\alpha) k_{i} z_{i}
$$

where $x_{i}$ is the displacement of the $i$ th storey (the node $i$ ); $k_{i}$ is the initial stiffness of the $i$ th storey; $\alpha$ is the post- to pre-yield stiffness ratio corresponding to $k_{i}$; in order to exhibit pinching phenomena, hysteretic displacement $z_{i}$ is proposed as the following ordinary differential equation [36]

$$
\dot{z}_{i}=p(\mathrm{z}) \frac{A \dot{x}_{i}-v\left(\beta\left|\dot{x}_{i}\right|\left|\dot{z}_{i}\right|^{n-1} z_{i}+\gamma \dot{x}_{i}\left|\dot{z}_{i}\right|^{n}\right)}{\eta}
$$

where $\dot{x}_{i}$ is the velocity of the $i$ th storey; $A$ describes the initial stiffness of the hysteretic component; $\beta$ and $\gamma$ are related to the strength of the member; $v$ and $\eta$ represent the strength degradation and stiffness degradation respectively, they can be expressed as

$$
v=1+\delta_{v} \varepsilon, \eta=1+\delta_{\eta} \varepsilon
$$

in which $\delta_{v}$ and $\delta_{\eta}$ are the degradation rate, $\varepsilon$ is the dissipated energy

$$
\varepsilon=\int_{0}^{t} z \dot{x} d t
$$

and

$$
p(\mathrm{z})=1.0-h_{1} e^{-\left[z \operatorname{sgn}(\dot{x})-q z_{\mathrm{u}}\right]^{2} / h_{2}^{2}}
$$

in which $\operatorname{sgn}(\cdot)$ is the signum function; $z_{\mathrm{u}}$ is the ultimate hysteretic component expressed as

$$
z_{\mathrm{u}}=\left(\frac{A / \eta}{v(\beta+\gamma)}\right)^{1 / n}
$$

and

$$
h_{1}(\varepsilon)=h_{s}\left(1-\mathrm{e}^{p \varepsilon}\right), \quad h_{2}(\varepsilon)=\left(\psi+\delta_{\psi} \varepsilon\right)\left(\lambda+h_{1}(\varepsilon)\right)
$$

where $h_{s}, p, q, \psi, \delta_{\psi}$ and $\lambda$ are the parameters of the Bouc-Wen model associated with pinching effects.

These 13 parameters in the Bouc-Wen model should be given before the model is employed in nonlinear dynamic analysis of the stochastic structures. Here the parameters proposed in reference [15] are adopted and shown in Tab. 3.

Table 3 The 13 parameters in the Bouc-Wen restoring model [15]

\begin{tabular}{cccccccc}
\hline Parameters & $\alpha$ & $\beta$ & $\gamma$ & $A$ & $n$ & $\delta_{v}$ & $\delta_{\eta}$ \\
\hline value & 0.04 & 30 & 10 & 1.0 & 1.0 & 2000 & 2000 \\
\hline Parameters & $h_{s}$ & $p$ & $q$ & $\psi$ & $\delta_{\psi}$ & $\lambda$ & \\
\hline value & 0.99 & 1000 & 0.25 & 0.05 & 5.0 & 0.5 \\
\hline
\end{tabular}

The curve of the restoring force changing against the inter-storey drift is presented in Fig. 8. 


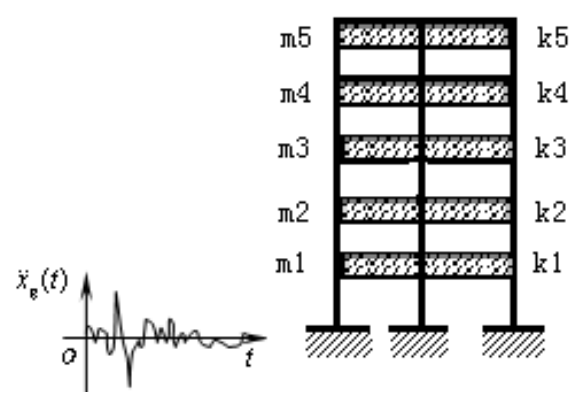

Fig.7 5-storey shear frame

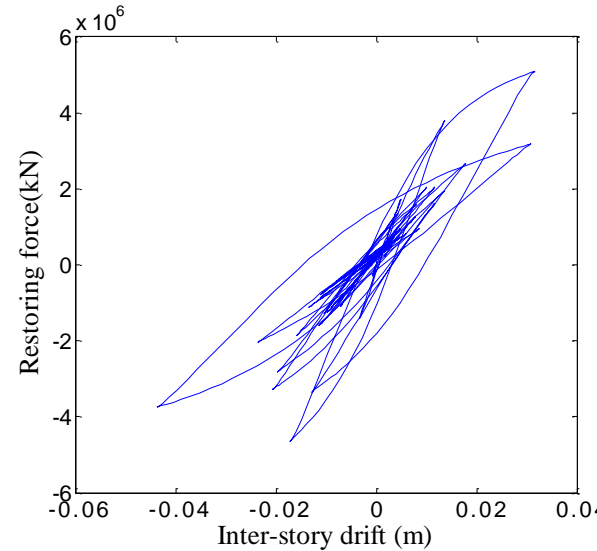

Fig. 8 Curve of restoring force and inter-storey drift

Seen from Fig. 8, the structure is at non-linear state. With the Bouc-Wen hysteretic model involved in the equation of motion, the dynamic responses of this structure become very complicated because of the coupling of randomness and nonlinearity. This problem can be solved expediently by incorporating PGI algorithm into the PDEM. The representative point set of the random-variate space is generated by PGI algorithm. There are 13587 points representing a 10-dimensional random-variate space according to the number theoretical method. As presented in section 5.2, these points are reduced to 247 points by the PGI algorithm. The stochastic dynamic responses of the five-storey and double span shear frame subjected to the earthquake excitation are investigated according to the procedures concluded in Part II of Sec. 4.4. Among them, for $\Theta=\theta_{q}$, solving Eq. (1) should be replaced by solving Eq. (20). The results based on the PDEM with 247 representative points determined by the PGI algorithm are compared with those obtained by Monte Carlo simulations of 9999 times (shown in Fig. 9), where PGI represents the results obtained by the PGI algorithm, MC represents the results obtained by the Monte Carlo simulation method.
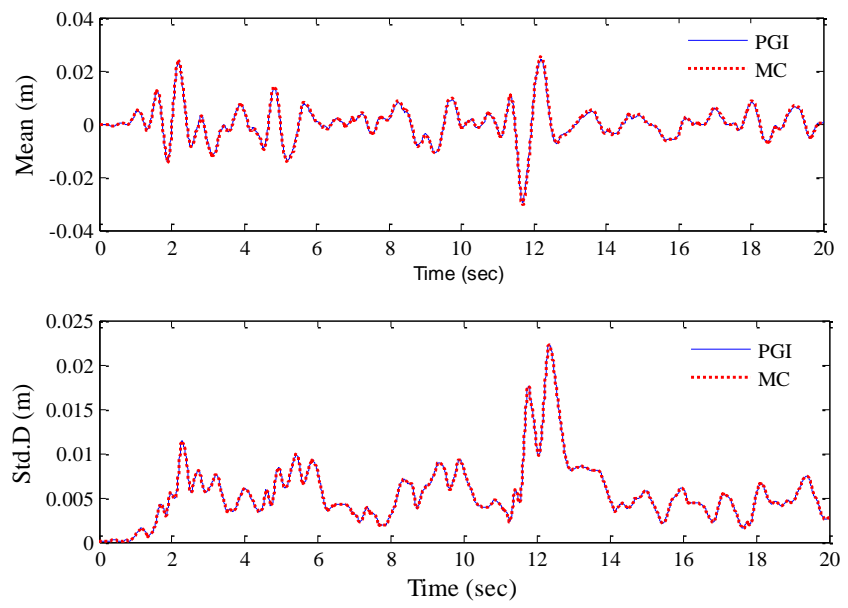

Fig.9 Comparison of the means and standard deviations between the PGI and the MC 
As shown in Fig. 9, the curves of means and standard deviations changing with time by incorporating the PGI algorithm into the PDEM basically coincide with those obtained by Monte Carlo simulation method. In general, the result obtained by Monte Carlo simulation method is considered more close to the exact solution. The errors between the results obtained by the two methods can be investigated quantitatively according to the 2-norm relative errors and the $\infty$-norm relative errors.

The 2-norm relative errors of means and standard deviations are defined as follows:

$$
\begin{aligned}
& e_{\|\mu\|_{2}}=\frac{\left\|\mu_{\mathrm{PGI}}(t)-\mu_{\mathrm{MC}}(t)\right\|_{2}}{\left\|\mu_{\mathrm{MC}}(t)\right\|_{2}} \\
& e_{\|\sigma\|_{2}}=\frac{\left\|\sigma_{\mathrm{PGI}}(t)-\sigma_{\mathrm{MC}}(t)\right\|_{2}}{\left\|\sigma_{\mathrm{MC}}(t)\right\|_{2}}
\end{aligned}
$$

where MC is the result obtained by the Monte Carlo simulation method, PGI is the result obtained by the PGI algorithm, $\|\cdot\|_{2}$ is the 2-norm, i.e. $\|s(t)\|_{2}=\sqrt{\sum_{i=1}^{k} s^{2}\left(t_{i}\right)}$.

According to Eqs (29) and (30), the 2-norm relative errors of means and standard deviations are $e_{\|\mu\|_{2}}=0.0344$ and $e_{\|\sigma\|_{2}}=0.0562$ respectively.

The $\infty$-norm relative errors of means and standard deviations are defined as follow:

$$
\begin{gathered}
e_{\|\mu\| \infty}=\frac{\left\|\mu_{\mathrm{PGI}}(t)-\mu_{\mathrm{MC}}(t)\right\|_{\infty}}{\left\|\mu_{\mathrm{MC}}(t)\right\|_{\infty}} \\
e_{\|\sigma\|_{\infty}}=\frac{\left\|\sigma_{\mathrm{PGI}}(t)-\sigma_{\mathrm{MC}}(t)\right\|_{\infty}}{\left\|\sigma_{\mathrm{MC}}(t)\right\|_{\infty}}
\end{gathered}
$$

where $\|\cdot\|_{\infty}$ is the $\infty$-norm, i.e. $\|s(t)\|_{\infty}=\max _{0<i<k}\left|s\left(t_{i}\right)\right|$.

The $\infty$-norm relative errors of means and standard deviations are $e_{\|\mu\|_{\infty}}=0.0269$ and $e_{\|\sigma\|_{\infty}}=0.0631$ respectively according to Eqs. (31) and (32).

The dynamic response of this stochastic structural system is a non-stationary stochastic process, seen from Fig. 9, because the standard deviations of displacements change obviously against time. A more visual method for estimating the results is to compare the probability density functions of displacement responses obtained by incorporating the PGI algorithm into the PDEM with those by the Monte Carlo simulation method. The PDF curves of displacement responses based on the PGI algorithm are compared with the histograms of the PDF of displacements obtained by the Monte Carlo simulations, as shown in Fig. 10, Fig. 11 and Fig. 12. The three figures present the displacement responses of the bottom storey at different time, 2.34 second, 11.88 second and 18.34 second (representing the initial stage, strong stage and attenuating stage of ground motion respectively).

The relative entropy is employed to describe the degree of approximation of the probability density curve obtained by incorporating the PGI algorithm into the PDEM and the probability density histogram obtained by the Monte Carlo simulation method. The relative entropy is defined as follows [33]: 


$$
\operatorname{En}\left(p_{X_{N}}, \phi\right)=\int_{-\infty}^{+\infty} \phi(x) \ln \frac{\phi(x)}{p_{X_{N}}(x)} d x
$$

where $\phi(x)$ is the probability density function obtained by the PDEM, $p_{X_{N}}(x)$ is the statistic result of the histogram obtained by the Monte Carlo simulation method.

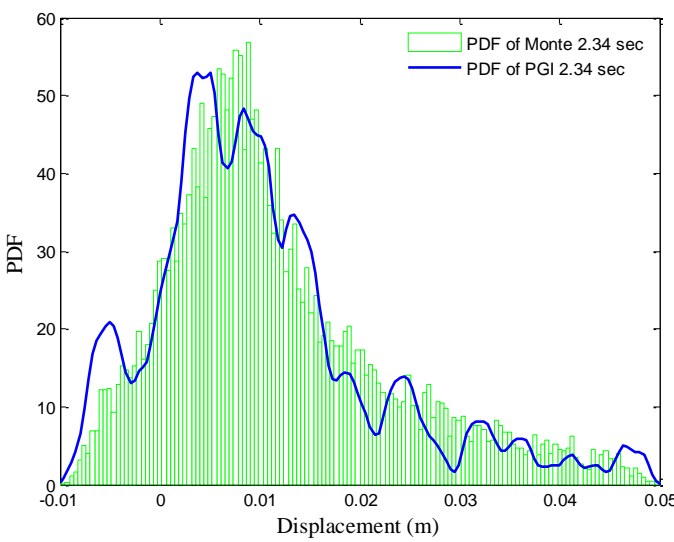

Fig.10 PDF of the displacement responses at 2.34 second

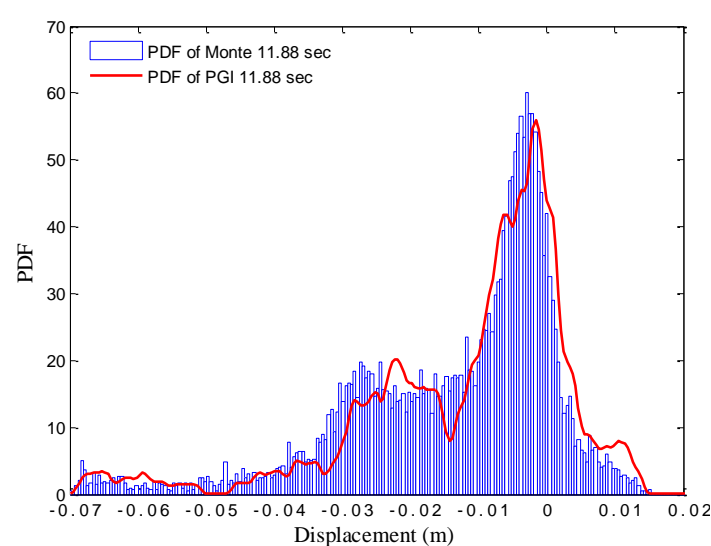

Fig.11 PDF of the displacement responses at 11.88 second

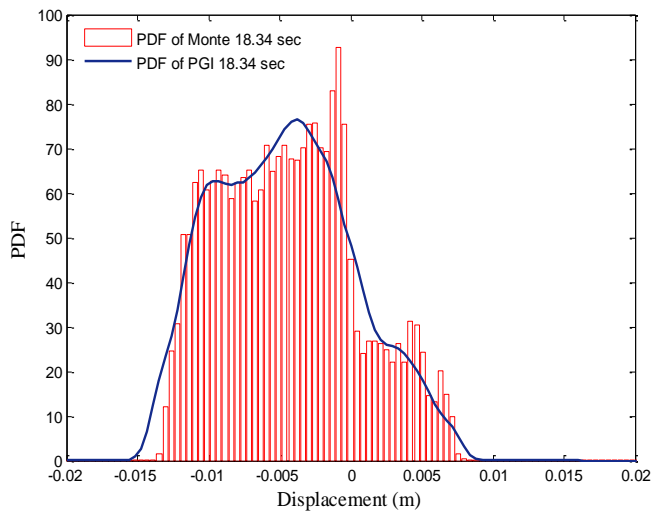

Fig.12 PDF of the displacement responses

at 18.34 second

If the relative entropy is close to zero, the curve of the former approximates to the histogram of the latter; otherwise, the higher the absolute value of the relative entropy, the bigger the gap between the curve and the histogram. The relative entropies at the different time are presented in Tab. 4.

Table 4 Relative entropies in different time

\begin{tabular}{cccc}
\hline & $\mathrm{t}=2.34 \mathrm{~s}$ & $\mathrm{t}=11.88 \mathrm{~s}$ & $\mathrm{t}=18.34 \mathrm{~s}$ \\
\hline relative entropies & 0.0614 & 0.0685 & 0.1402 \\
\hline
\end{tabular}

It is seen from the above results that the nonlinear dynamic analysis of the stochastic structural system by incorporating the algorithm PGI into the PDEM has enough accuracy. Meanwhile the 
computational burden is reduced effectively through the decrease of the number of representative points.

\section{Conclusions}

An original approach is proposed for identifying point groups in an initial point set of a random-variate space. The ideas of clustering in the field of data mining and the graph theory are first introduced into the stochastic dynamic analysis. According to the density criterion and the partition of space, the restraint condition of the remaining subspace is imposed on the identification of point groups to lower the GF-discrepancy of the representative point set. The stochastic nonlinear dynamic responses can be studied comprehensively by incorporating the proposed algorithm into the PDEM. A 5-storey and double span shear frame with the random structural parameters is investigated. Some characteristics of the responses are observed and discussed. In order to compare the computational properties and the accuracy, the Monte Carlo simulations are carried out. It is found that the proposed algorithm can improve the computational efficiency by reducing the number of the representative points effectively, and ensure the computational accuracy of the dynamic analysis of stochastic systems. The further research will focus on the PGI algorithm used in higher-dimensional random-variate space.

\section{Acknowledgement}

The support from the Natural Science Foundation for Major International Cooperation Project of China (Grant No. 5126112037), the National Science Foundation for Integration Project of China (Grant No. 91315301) and the National Science Foundation for General Project of China (Grant No. 51378377) are gratefully acknowledged.

\section{References}

[1] J. Li, Stochastic Structural Systems - Analysis and Modeling, Science Press, Beijing, 1996.

[2] J.B. Roberts, P.D. Spanos, Random Vibration and Statistical Linearization, John Wiley and Sons, Chichester, 1990.

[3] J. Li, J.B. Chen, General probability density evolution equation for stochastic dynamic systems, Chinese Journal of Natural Science Development, 6 (2006) 712-719.

[4] L.D. Lutes, S. Sarkani, Random Vibration: Analysis of Structural and Mechanical Systems, Elsevier, Amsterdam, 2004.

[5] T. Fang, Random Vibration of Engineering, National Defence Industry Press, Beijing, 1995.

[6] W.Q. Zhu, Random Vibration, Science Press, Beijing, 1992.

[7] R.G. Ghanem, P.D. Spanos, Stochastic finite elements: a spectral approach, Springer - Verlag, Berlin, 1991.

[8] D.B. Xiu, G.E. Karniadakis, The Wiener-Askey polynomial chaos for stochastic differential equations, SIAM Journal on Scientific Computing 242 (2) (2002) 619-644.

[9] X.L. Wan, G.E. Karniadakis, Multi-element generalized polynomial chaos for arbitrary probability measures, SIAM Journal on Scientific Computing 28 (3) (2006) 901-928. 
[10] D.B. Xiu, J.S. Hesthaven, High-order collocation methods for differential equations with random inputs, SIAM Journal on Scientific Computing 27 (3) (2005) 1118-1139.

[11] J. Foo, X.L. Wan, G.E. Karniadakis, The multi-element probabilistic collocation method (ME-PCM): error analysis and application, Journal of computational Physics 227 (2008) 9572-9595.

[12] J. Li, J.B. Chen, Probability density evolution method for dynamic response analysis of structures with uncertain parameters, Computational Mechanics 34 (2004) 400-409.

[13] J. Li, J.B. Chen, The probability density evolution method for dynamic response analysis of non-linear stochastic structures, International Journal for Numerical Methods in Engineering 65 (2005) 882-903.

[14] J.B. Chen, J. Li, Dynamic response and reliability analysis of nonlinear stochastic structures, Probabilistic Engineering Mechanics 20 (1) (2006) 33-44.

[15] J.B. Chen, J. Li, Stochastic seismic response analysis of structures exhibiting high nonlinearity, Computers and Structures 88 (2010) 395-412.

[16] J. Li, J.B. Chen, W.L. Sun, Y.B. Peng, Advances of the probability density evolution method for nonlinear stochastic systems, Probabilistic Engineering Mechanics 28 (2012) 132-142.

[17] J.B. Chen, R. Ghanem, J. Li, Partition of the probability-assigned space in probability density evolution analysis of nonlinear stochastic structures, Probabilistic Engineering Mechanics 24 (2009) 27-42.

[18] J. Li. J.B. Chen, The number theoretical method in response analysis of nonlinear stochastic structures, Computational Mechanics 39 (2007) 693-708.

[19] J.B. Chen, J. Li, Strategy for selecting representative points via tangent spheres in the probability density evolution method, International Journal for Numerical Methods in Engineering 74 (2008) 1988-2014

[20] N. Victoir, Asymmetric cubature formulae with few points in high dimension for symmetric measures, SIAM Journal on Numerical Analysis 42 (2004) 209-227.

[21] M. Shinozuka, C.M. Jan, Digital simulation of random process and its applications, Journal of Sound and Vibration 25 (1) (1972) 111-128.

[22] S.H. Zhang, PDEM-based high efficient dynamic response analysis and reliability evaluation for nonlinear structures, Master Thesis, Tongji University, 2013.

[23] R. Agrawal, J. Gehrke, D. Gunopulos, P. Raghavan, Automatic subspace clustering of high dimensional data for data mining applications, Proceedings of ACM SIGMOD, Seattle, Washington, USA, 1998, pp. 94-105.

[24] Y. Li, C. Campbell, M. Tipping, Bayesian automatic relevance determination algorithms for classifying gene expression data, Bioinformatics 18 (10) (2002) 1332-1339.

[25] O. Zamir, O. Etzioni, Web document clustering: a feasibility demonstration, Proceedings of the 19th Annual International ACM SIGIR Conference on Research and Development in Information Retrieval, 1998, pp. 46-54.

[26] L. Wei, X.Q. Gong, W.N. Qian, O.Y. Zhou, Finding outliers in high dimensional space, Chinese Journal of Software 13 (2) (2002) 280-290. 
[27] J. Li, J.B. Chen, Stochastic Dynamics of Structures, John Willey and Sons, Singapore, 2009.

[28] L.G. Hua, Y. Wang, Application of Number Theory in the Approximate Analysis, Science Press, Beijing, 1978.

[29] J.B. Chen, S.H. Zhang, J. Li, Improving point selection in cubature by a new discrepancy, SIAM Journal on Scientific Computing 35 (5) (2014) A2121-A2149.

[30] C.C. Aggarwal, A. Hinneberg, D.A. Kein, On the surprising behavior of distance metrics in high dimensional space, In Proceedings of the International Conference on Database Theory, London, Britain, 2001, pp. 420-434.

[31] Z.J. Liu, Research on the orthogonal expansion theory and its application of the stochastic dynamic action in engineering, PhD Thesis, Tongji University, 2006.

[32] L.Y. Miao, C.Q. Wang, Graph Theory and Its Algorithm, China Mining University Press, Xuzhou, 2012.

[33] Y. Ohsaki, On the significance of phase content in earthquake ground motion, Earthquake Engineering and Structural Dynamics 7 (1979) 427-439.

[34] M. Loeve, Probability Theory, Springer-Verlag, Berlin, 1977.

[35] Y.K. Wen, Method for random vibration of hysteretic systems, Journal of the Engineering Mechanics Division 102 (2) (1976) 249-263.

[36] T.T. Baber, M.N. Noori, Random vibration of degrading, pinching systems, Journal of Engineering Mechanics 111 (8) (1085) 1010-1027.

[37] R. Agrawal, R. Srikant, Fast algorithms for mining association rules in large databases, Proceedings of the 20th International Conference on Very Large Data Bases (VLDB'94), (1994) 487-499.

[38] R. N. Bracewell, The Harley Transform, Oxford University Press, New York, 1986.

[39] F. Aurenhammer, Voronoi Diagrams - A Survey of a Fundamental Geometric Data Structure, ACM Computing Surveys 23(3) (1991) 345-405.

[40] J. Li, Z.J. Liu, Decomposition of stochastic process based on standard orthogonal basis, Journal of Tongji University 34(10) (2006) 1279-1283.

\section{Figure captions}

Fig. 1: First 50 eigenvalues of the correlation matrix. Eigenvalues of the correlation matrix are plotted in this figure. A trend of eigenvalues changing with their orders is presented.

Fig. 2: Projection of points in the remaining 1-dimensional subspace. The filled circles with different colors on $\mathrm{x}-\mathrm{y}$ plane represent the 2-dimesion projections of some points in the 3-dimension space. The hollow circles with different colors on z-axis represent the remaining 1-dimension projection of these points. 
Fig. 3: Scattering of 80 points generated by pseudo random number method. The scattering of 80 points in a 2-dimensional random-variate space generated by the pseudo random number method are plotted in the figure. Regions bounded by the blue lines are the Voronoi regions represented by these points.

Fig. 4: Scattering of 38 representative points generated by the PGI algorithm. The scattering of 38 points generated by the PGI algorithm are plotted in the figure.

Fig. 5: Scattering of 38 points generated by pseudo random number method. The scattering of 38 points generated by the pseudo random number method are plotted in the figure.

Fig. 6: Scattering of the points in the 2-dimensional number theoretical point set. The scattering of 38 points generated by the number theoretical method are plotted in the figure.

Fig. 7: 5-storey shear frame. The curve in the bottom left corner of the figure is the acceleration curve of the earthquake excitation.

Fig. 8: Curve of restoring force and inter-storey drift.

Fig. 9: Comparison of the means and standard deviations between the PGI algorithm and the MC method. The blue line represents the results obtained by the PGI algorithm. The dotted red line represents the results obtained by the Monte Carlo method.

Fig. 10: PDF of the displacement responses at 2.34 second. The blue line represents the results obtained by the PGI algorithm. The green hollow columns represent the results obtained by the Monte Carlo method.

Fig. 11: PDF of the displacement responses at 11.88 second. The red line represents the results obtained by the PGI algorithm. The blue hollow columns represent the results obtained by the Monte Carlo method.

Fig. 12: PDF of the displacement responses at 18.34 second. The blue line represents the results obtained by the PGI algorithm. The red hollow columns represent the results obtained by the Monte Carlo method. 
FIGURES

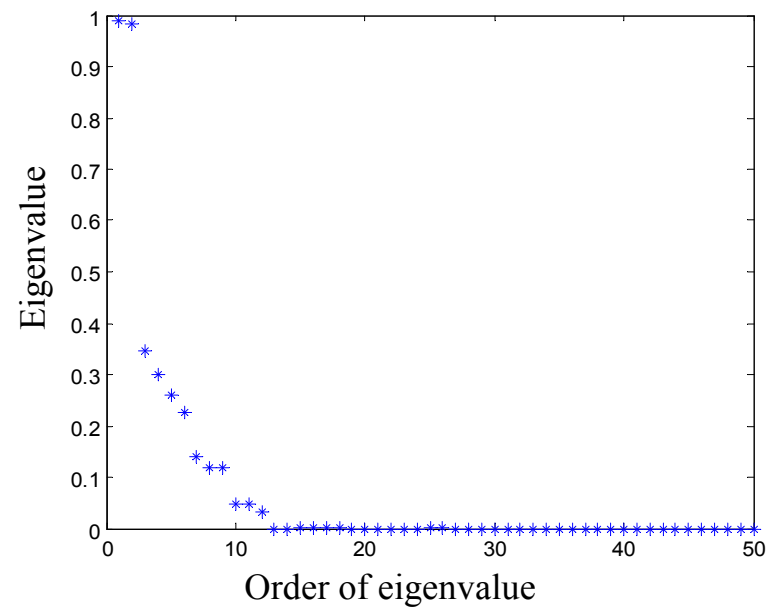

Fig. 1 First 50 eigenvalues of the correlation matrix 


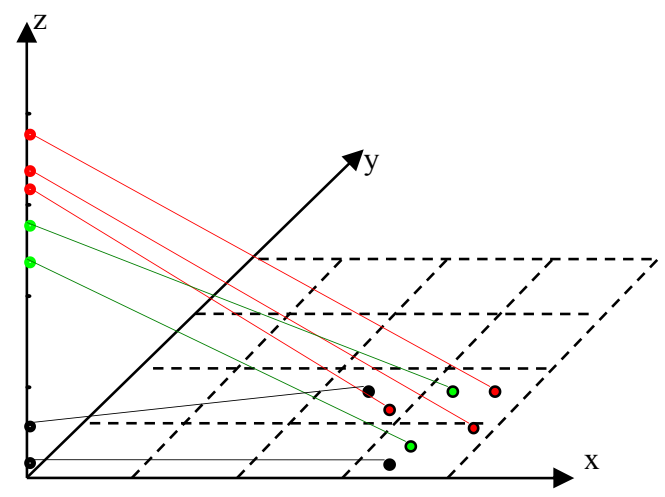

Fig. 2 Projection of points in the remaining 1-dimensional subspace 


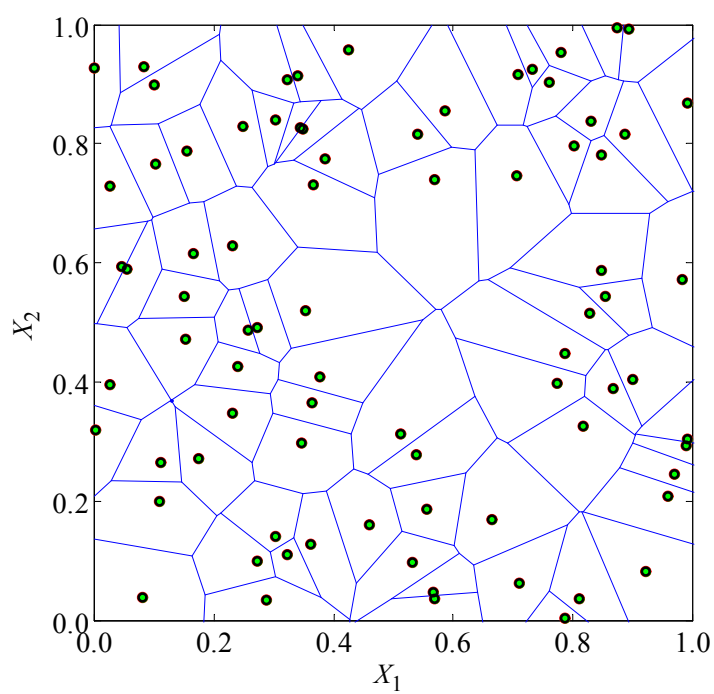

Fig. 3 Scattering of 80 points generated by pseudo random number method 


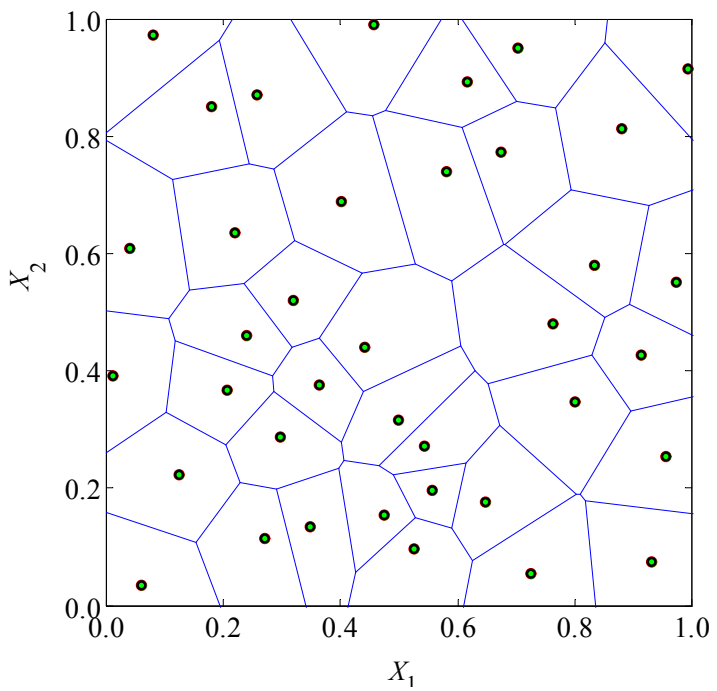

Fig. 4 Scattering of 38 representative points generated by the PGI algorithm 


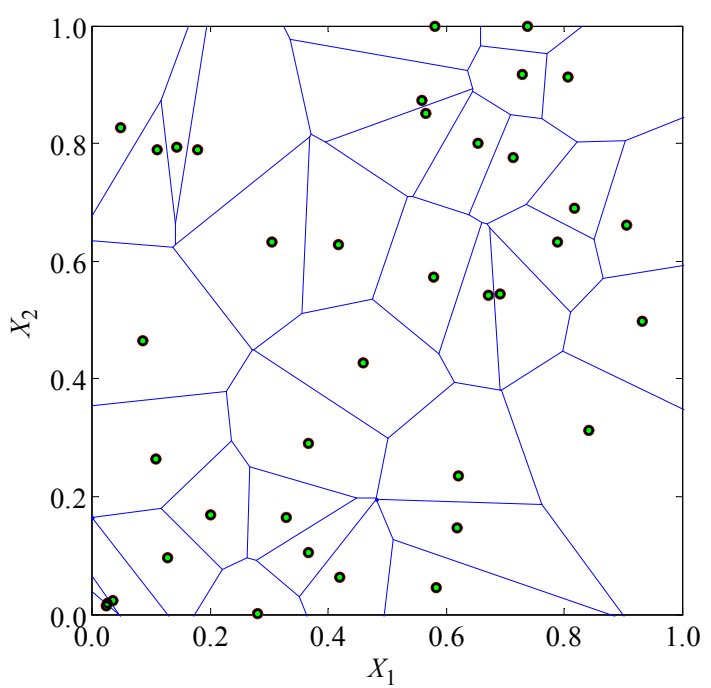

Fig. 5 Scattering of 38 points generated by pseudo random number method 


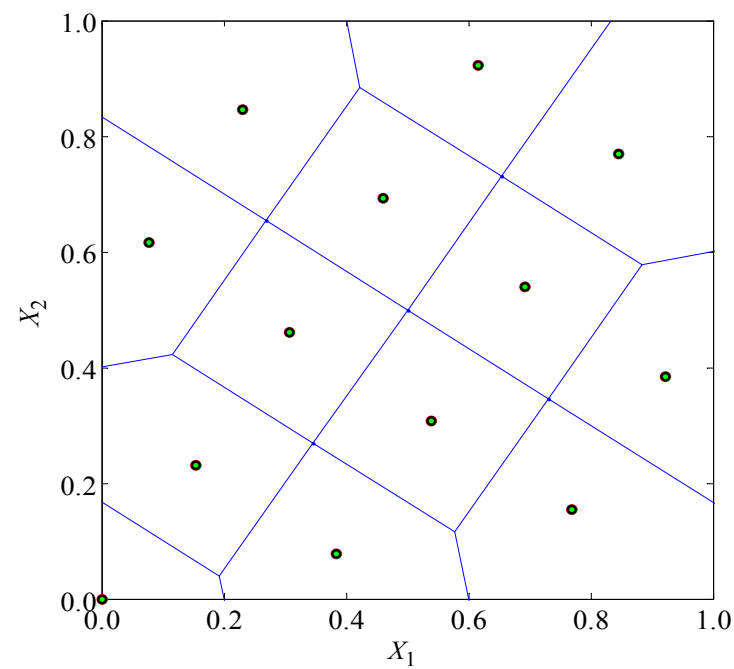

Fig. 6 Scattering of the points in the 2-dimensional number theoretical point set 


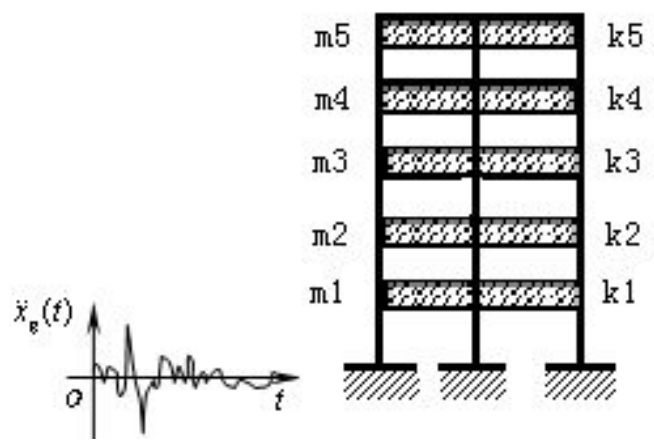

Fig.7 5-storey shear frame 


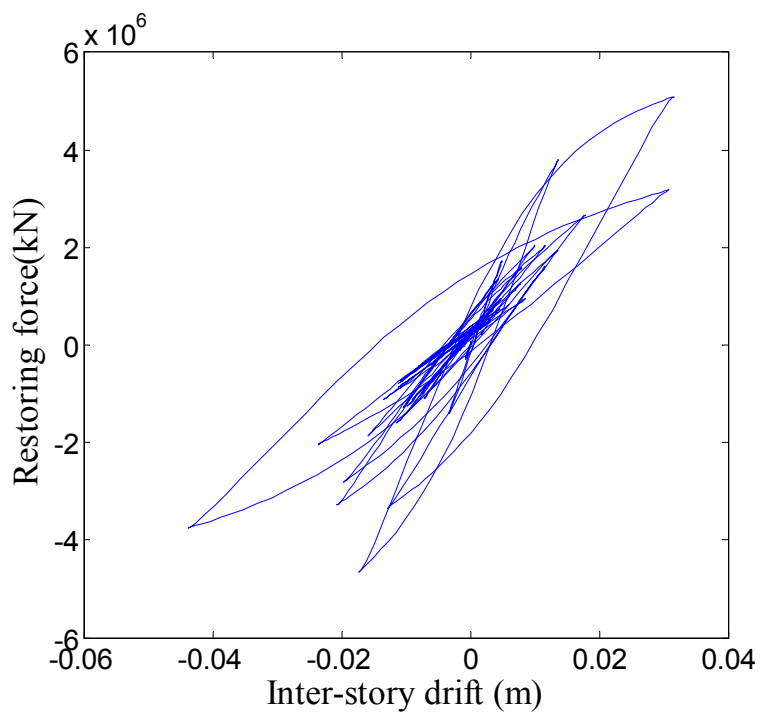

Fig. 8 Curve of restoring force and inter-storey drift 

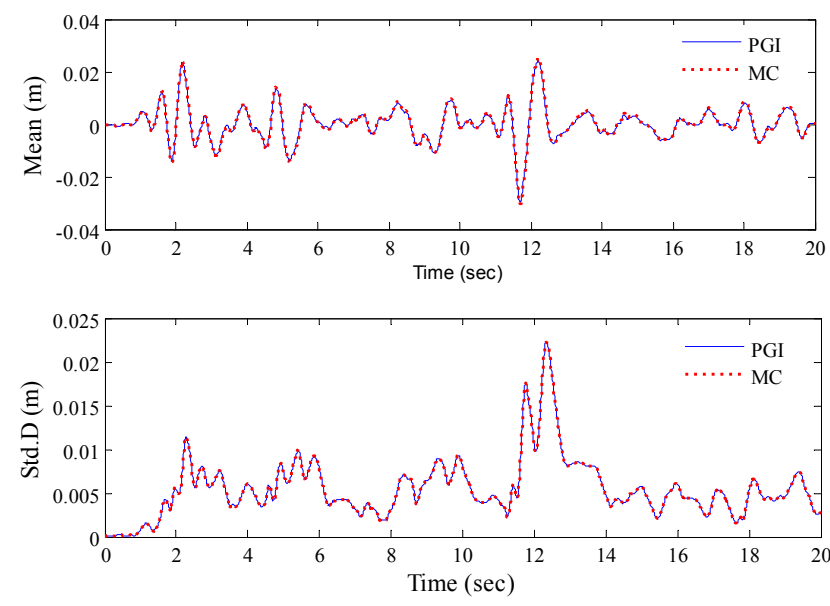

Fig.9 Comparison of the means and standard deviations between the PGI and the MC 


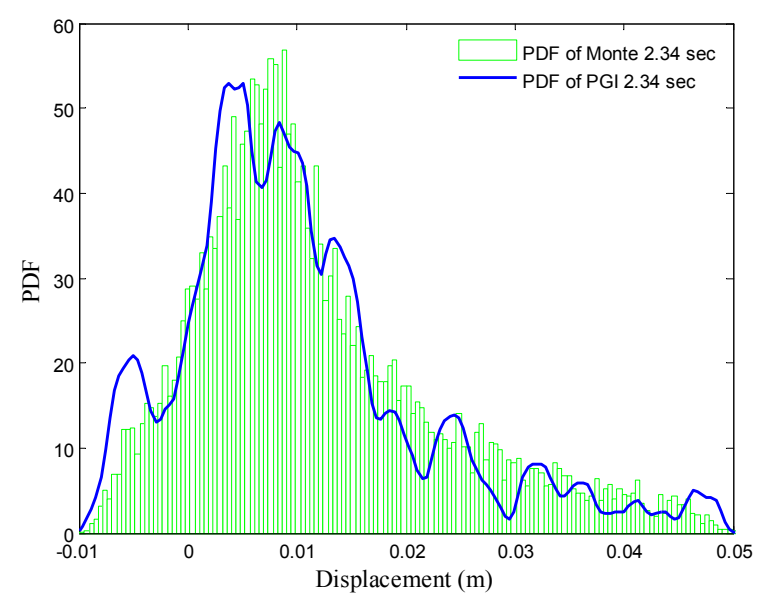

Fig.10 PDF of the displacement responses at 2.34 second 


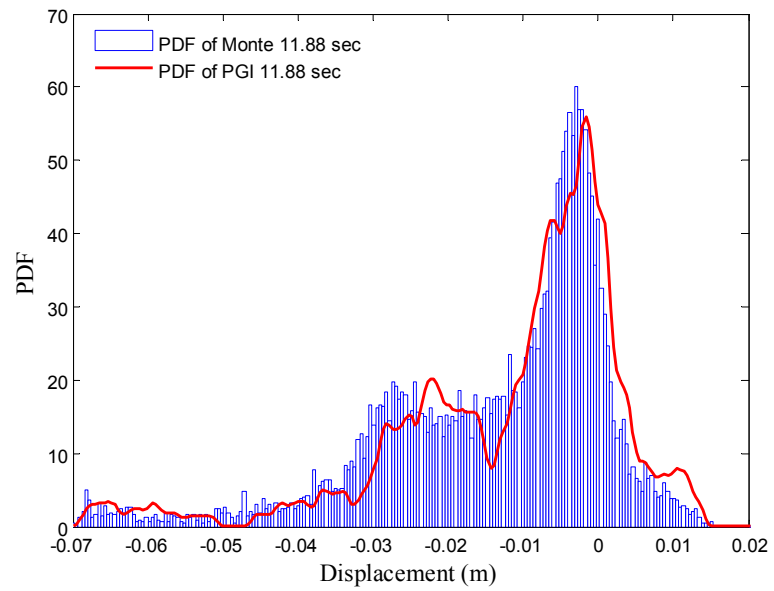

Fig.11 PDF of the displacement responses at 11.88 second 


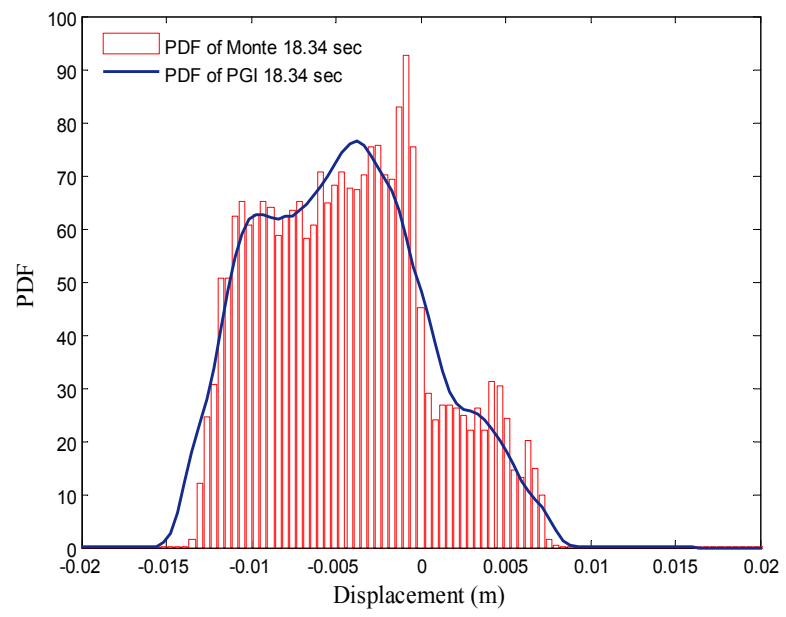

Fig.12 PDF of the displacement responses at 18.34 second 
Table 1 Discrepancies of three point sets in the 2-dimensional space

\begin{tabular}{ccccc}
\hline Case & Item & $\begin{array}{c}\text { PRN_80 } \\
\text { point set }\end{array}$ & $\begin{array}{c}\text { PGI } \\
\text { point set }\end{array}$ & $\begin{array}{c}\text { PRN_38 } \\
\text { point set }\end{array}$ \\
\hline $\begin{array}{c}\text { 2-dimensional } \\
\text { space }\end{array}$ & Point number & 80 & 38 & 38 \\
\hline
\end{tabular}

Table 2 Discrepancies of three kinds of point sets in the 10-dimensional space

\begin{tabular}{ccccc} 
Case & Item & PGI & NTM & PRN \\
\hline $\begin{array}{c}\text { 10-dimesional } \\
\text { space }\end{array}$ & Point number & 247 & 248 & 247 \\
\hline
\end{tabular}

Table 3 Relative entropies in different time

\begin{tabular}{cccc}
\hline & $\mathrm{t}=2.34 \mathrm{~s}$ & $\mathrm{t}=11.88 \mathrm{~s}$ & $\mathrm{t}=18.34 \mathrm{~s}$ \\
\hline relative entropies & 0.0614 & 0.0685 & 0.1402 \\
\hline
\end{tabular}

